\title{
On Optimal Agendas for Multi-Issue Negotiation
}

\author{
Shaheen Fatima1 Michael Wooldridge2 Nicholas R. Jennings3 \\ 1 Department of Computer Science \\ Loughborough University, Loghborough, LE11 3TU, U.K. \\ S.S.Fatima@lboro.ac.uk \\ 2 Department of Computer Science \\ University of Liverpool, Liverpool, L69 7ZF, U.K. \\ mjweliv.ac.uk \\ 3 School of Electronics and Computer Science \\ University of Southampton, Southampton SO17 1BJ, U.K. \\ nrjeecs.soton.ac.uk
}

\begin{abstract}
This paper analyzes bilateral multi-issue negotiation where the issues are divisible, there are time constraints in the form of deadlines and discount factors, and the agents have different preferences over the issues. The issues are negotiated using the package deal procedure. The set of issues to be negotiated is a choice variable in that the agents can decide what issues to negotiate. This set is called the negotiation agenda. Since the outcome of negotiation depends on the agenda, it is important to determine what agenda maximizes an agent's utility and is therefore its optimal agenda. To this end, this paper presents polynomial time methods for finding an agent's optimal agenda.
\end{abstract}

\section{Introduction}

Negotiation has long been studied by economists and game theorists but is now receiving increasing attention from researchers in multi-agent systems $[10,13]$. In the existing work, the analysis of negotiation typically begins with a given set of issues and the parties' preferences (in the form of their utilities) for different possible settlements of the issues. Within this framework, theorists have investigated a range of negotiation procedures such as the package deal procedure (PDP), the simultaneous procedure (SP), and the sequential procedure (SQP) $[7,8,2]$.

It is well known that different procedures result in different outcomes and, therefore, give different utilities to the agents [6]. So it is important that agents choose the right procedure. Moreover, for a given procedure, it is also important that the agents choose an appropriate agenda. The term agenda refers to the set of issues included for negotiation [11]. The agenda is important because, irrespective of the procedure, the outcome of negotiation depends on the agenda [7,14]. Thus, given the utility maximizing feature of agents, it is important to find what agenda maximizes their utility and is therefore optimal for them. For example, consider a car dealer who has five second hand cars to sell. A potential buyer may be interested in buying two of these. So he must first choose which two cars to negotiate the price for (i.e., from all possible subsets of size 
two, he must choose the one that maximizes his utility and so is his optimal agenda). Note that here, the buyer has choice over the agenda but not the seller.

Although the importance of agendas has been recognized [7, 11], most existing work has taken the agenda as given and then analyzed the outcome for different procedures $[8,5,6]$. But as the above example illustrates, the set of issues to be negotiated themselves are often choice variables (i.e., what issues to negotiate can be chosen by a negotiator) whose ultimate configuration can have decisive effects on the negotiation outcome [14]. Thus, in addition to knowing what procedure is best for an agent, it is important to know what agenda maximizes an agent's utility and is therefore its optimal agenda. To this end, this paper analyzes the problem of finding optimal agendas in the context of the PDP (future work will deal with SP and SQP). The key contribution of this paper lies in presenting, for the first time, polynomial time methods for determining optimal agendas for the PDP.

The rest of the paper is structured as follows. Section 2 provides a discussion of related literature. Section 3 describes the setting and defines 'agenda' and 'optimal agenda'. Section 4 shows how to find optimal agendas for the complete information setting. Section 5 builds on Section 4 to show how to find optimal agendas in an incomplete information setting. Section 6 concludes.

\section{Related Literature}

There are different procedures such as the PDP, the SP, and the SQP for multi-issue negotiation, and the term agenda has different meanings in these different contexts. For the PDP (which is the focus of this paper) and the SP, the term agenda refers to the set of issues to be included for negotiation. But for the SQP, the term refers to not just the set of issues to be included for negotiation but also the order in which they will be negotiated.

Although the importance of agendas has been recognized [7, 11], most existing work has taken the set of issues as given and then analyzed the equilibrium outcome for different procedures $[9,8,6]$. In the context of the SQP, [1] takes the set of issues to be negotiated as given and provides an analysis of the role of information and time preferences on the equilibrium, while $[4,5]$ has dealt with taking the set of issues to be negotiated as given and finding an optimal ordering for the given set. But as the example outlined in the Introduction illustrates, the set of issues to be negotiated themselves are often choice variables (i.e., what issues to negotiate can be chosen by a negotiator) whose ultimate configuration can have decisive effects on the negotiation outcome [14]. Thus, a negotiator must make three key choices: he/she must decide the following:

1. what negotiation procedure to use,

2. what set of issues to negotiate, and

3. for the SQP, what ordering to use for negotiating a given set of issues.

To our knowledge, there is no existing work that deals with finding what set of issues to negotiate. Hence, the novelty of this paper lies in showing how to find the set of issues to negotiate. Specifically, it analyzes the problem of finding optimal agendas in the context of the PDP. The key contribution of this paper lies in presenting, for the first time, polynomial time methods for determining optimal agendas for the PDP. 


\section{The Negotiation Setting}

An agent's optimal agenda depends on its equilibrium utility from all possible agendas. So we first give an overview of the equilibrium for single and multi-issue negotiation. On the basis of this equilibrium, we will show how to find optimal agendas in Sections 4 and 5. A formal definition of optimal agenda is given toward the end of this section.

\subsection{Equilibrium for Single Issue}

We use the single issue model of [6] in which two agents, $a$ and $b$, negotiate over a single divisible issue which is a 'pie' of size 1 . The agents want to determine how to split it between themselves. Let $n \in N^{+}$be the deadline and $0<\delta \leq 1$ the discount factor for both agents. The agents use an alternating offers protocol [12], which proceeds through a series of time periods. One of the agents, say $a$, starts in the first time period (i.e., $t=1)$ by making an offer $\left(0 \leq x^{a} \leq 1\right)$ to $b$. Agent $b$ can accept/reject the offer. If it accepts, negotiation ends in an agreement with $a$ getting $x^{a}$ and $b$ getting $x^{b}=1-x^{a}$. Otherwise, negotiation goes to the next time period, in which $b$ makes a counter-offer. This process continues until one of the agents either accepts an offer or quits negotiation (resulting in a conflict).

Agent $a$ 's utility at time $t$ from a share $x^{a}$ is $u^{a}\left(x^{a}, t\right)=x^{a} \delta^{t-1}$ if $t \leq n$, otherwise it is zero. For $b, u^{b}$ is analogous. For this setting, the equilibrium offers are obtained using backward induction (BI) as follows. Let $n=1$ and let $a$ be the first mover. If $b$ accepts $a$ 's proposal at $t=1$, the division occurs as agreed; if not, neither agent gets anything (since the deadline is $n=1$ ). Here, $a$ is in a powerful position and is able to keep $100 \%$ of the pie and give nothing to $b^{1}$. Agent $b$ accepts and agreement takes place at $t=1$.

Now, let $n=2$ and $\delta=1 / 2$. The first mover (say $a$ ) decides what to offer at $t=1$, by looking ahead to $t=2$ and reasoning backwards. Agent $a$ reasons that if negotiation goes to $t=2, b$ will take $100 \%$ of the shrunken pie by offering $[0,1 / 2]$. So, at $t=1$, if $a$ offers $b$ anything less than $1 / 2, b$ will reject the offer. So, at $t=1, a$ offers $[1 / 2,1 / 2]$. Agent $b$ accepts and an agreement occurs at $t=1$. In this way, BI was used to obtain the equilibrium offer for $t>2$.

\subsection{Equilibrium for Multiple Issues for the PDP}

Let $I=\{1,2, \ldots, m\}$ be the set of issues. Agent $a$ 's (b's) preference for issue $i$ is represented with a weight $w_{i}^{a} \in R_{+}\left(w_{i}^{b} \in R_{+}\right)$. Each issue is a pie of size 1 and can be split between the agents such that if $x_{i}^{a}$ and $x_{i}^{b}$ are $a$ 's and $b$ 's shares for issue $i$, then $x_{i}^{a}+x_{i}^{b}=1$. Here $n$ is the deadline for all the issues, and $\delta$ the discount factor for all of them. Agent $a$ 's cumulative utility at time $t \leq n$ is given by $U^{a}\left(I, x^{a}, t\right)=$ $\delta^{t-1} \sum_{i=1}^{m} w_{i}^{a} x_{i}^{a}$. For $b, U^{b}\left(I, x^{b}, t\right)$ is analogous. As before, an agent's utility for $t>n$ is zero.

\footnotetext{
${ }^{1}$ It is possible that $b$ may reject such a proposal. But, irrespective of whether $b$ accepts or rejects, it gets zero utility (since the deadline is $n=1$ ). So, $b$ accepts $a$ 's offer.
} 
For the above model, the equilibrium was obtained, using BI, as follows [6]. We give an overview of this first for complete information setting, and then explain its extension to an incomplete information setting.

The complete information setting. Let $\mathrm{SA}(t)$ ( $\mathrm{SB}(t))$ denote $a$ 's $(b$ 's) equilibrium strategy for time $t$. For $t=n$, the offering agent gets a $100 \%$ of all the shrunken pies. For all previous time periods, $t<n$, the offering agent (say $a$ ) proposes a package $\left(\left[x^{a}, x^{b}\right]\right)$ such that $b$ 's cumulative utility from it is what $b$ would get from its own offer for $t+1$. If there is more than one such package, then $a$ must choose the one that maximizes its own cumulative utility. Thus, $a$ must solve the following trade-off problem (called TA): maximize $\sum_{i=1}^{m} w_{i}^{a} x_{i}^{a}$, subject to $\sum_{i=1}^{m} w_{i}^{b}\left(1-x_{i}^{a}\right)=Y$ where $0 \leq x_{i}^{a} \leq 1$. Here, $Y$ is $b$ 's cumulative utility from its own offer $\mathrm{SB}(\mathrm{t}+1)$. On the other hand, if $a$ receives an offer $\left[x^{a}, x^{b}\right]$ at time $t$, then it accepts if $U^{a}\left(I, x^{a}, t\right)=Z$ where $Z$ is $a$ 's cumulative utility from its own offer $\mathrm{SA}(t+1)$. The equilibrium strategy for $b$ (in terms of TB) is analogous. Thus we have:

$$
\mathrm{SA}(n)= \begin{cases}\text { OFFER }[\mathbf{1 , 0}] & \text { If } a \text { 's turn to offer } \\ \text { ACCEPT } & \text { If } a \text { 's turn to receive }\end{cases}
$$

where $\mathbf{0}$ (1) denotes a vector of $m$ zeros (ones). For all preceding time periods $t<n$, the strategies are as follows:

$$
\mathrm{SA}(t)= \begin{cases}\text { OFFER TA } & \text { If } a \text { 's turn to offer } \\ \text { If }\left(U^{a}\left(I, x^{a}, t\right) \geq Z\right) \text { ACCEPT If } a \text { receives } x^{a} \\ \text { else REJECT }\end{cases}
$$

For $b, \mathrm{SB}(\mathrm{t})$ is analogous. Both TA and TB are standard fractional knapsack problems [3]. The solution to TA is for $a$ to consider issues in ascending order of $w_{i}^{a} / w_{i}^{b}$ and allocate to $b$ maximum possible share for the individual issues until $b$ 's cumulative utility equals $Y$. If the issues $1, \ldots, m$ are in ascending order of $w_{i}^{a} / w_{i}^{b}$, the equilibrium solution is $x^{a}=\left\{0, . ., 0, x_{c}^{a}, 1, . ., 1\right\}, x^{b}=\left\{1, . ., 1,1-x_{c}^{a}, 0, . ., 0\right\}$. The solution to TB is analogous.

An incomplete information setting. Here, the agents are uncertain about their utilities. Let $T$ be the number of possible utility function pairs. The $j$ th possible pair $\left(U_{j}^{a}, U_{j}^{b}\right)$ occurs with probability $\gamma_{j}$. For $a$, the $j$ th function is: $U_{j}^{a}\left(I, x^{a}, t\right)=\delta^{t-1} \sum_{i=1}^{m} w_{i j}^{a} x_{i}^{a}$, and its expected utility is $E U^{a}\left(I, x^{a}, t\right)=\delta^{t-1} \sum_{j=1}^{T} \gamma_{j} \times U_{j}^{a}\left(I, x^{a}, t\right)$. Agent $a$ 's expected weight for issue $i$ is $e w_{i}^{a}=\delta^{t-1} \sum_{j=1}^{T} \gamma_{j} w_{i j}^{a}$. For $b, U_{j}^{b}, E U^{b}\left(I, x^{b}, t\right)$, and $e w_{i}^{b}$ are analogous.

Given this, agent $a$ 's tradeoff problem at time $t$ is to find a package $\left[x^{a}, x^{b}\right]$ that solves the following problem:

$$
\begin{aligned}
\text { TA-I maximize } & E U^{a}\left(I, x^{a}, t\right) \\
\text { subject to } & E U^{b}\left(I, x^{b}, t\right)=E Y \quad 0 \leq x_{i}^{a} \leq 1
\end{aligned}
$$

Here $I$ is fixed and $E Y$ is $b$ 's equilibrium utility for $t+1$. Given this, $a$ 's equilibrium strategy for time $t$ is the same as SA(t) (defined earlier) with TA replaced with TA-I, and 
$U^{a}$ replaced with $E U^{a}$. Likewise for agent $b$. The problem TA-I is also the standard fractional knapsack problem, and is solvable using a greedy approach.

On the basis of the above equilibrium, we will show how to find an optimal agenda, for the PDP, for each agent.

\subsection{The Negotiation Agenda}

As noted in Section 1, in many cases, the set of issues to be negotiated are choice variables. So, before negotiation begins, the agents must decide upon an agenda which we define as follows:

Definition 1. In the context of the PDP, an agenda $A^{g}$ of size $g \leq m$ is a set of $g$ issues, i.e., $A^{g} \subseteq I$ such that $\left|A^{g}\right|=g$. Let $A G^{g}$ denote the set of all possible agendas of size g.

Definition 2. For the PDP, an agenda $\left(A A^{g}\right)$ of size $g \leq m$ is agent a's optimal agenda if

$$
A A^{g}=\arg \max _{X \in A G^{g}} E U^{a}\left(X, x^{a}, 1\right)
$$

where $x^{a}$ denotes a's equilibrium allocation (for agenda $X$, for $t=1$ ). For $b, A B^{g}$ is analogous. For the complete information setting, $E U^{a}$ and $E U^{b}$ are replaced with $U^{a}$ and $U^{b}$ respectively.

For the agenda $I$ containing $m$ issues, Section 3.2 showed how to find equilibrium outcomes (i.e., $U^{a}, E U^{a}, U^{b}$, and $E U^{b}$ ). Given this equilibrium, our problem now is to find each agent's optimal agenda: $A A^{g}$ and $A B^{g}$ for $1 \leq g \leq m$. We show how to find these agendas in Section 4.

Below, we focus on the case where one of the agents, say $a$, prefers different issues differently but $b$ prefers all the issues equally ${ }^{2}$. So, for $a$, different issues have different weights but $b$ has the same ${ }^{3}$ weight for all the issues. We let the issues $\{1, \ldots, m\}$ be such that $w_{i}^{a} \leq w_{i+1}^{a}$ for $1 \leq i \leq n-1$. Also, for agenda $X \in A G^{g}$, we let $X=\{1, \ldots, g\}$ be such that $w_{X_{i}}^{a} \leq w_{X_{i+1}}^{a}$ for $1 \leq i<g$. Note that, although we are viewing $X$ as a list, as per its definition, an agenda is a set because the equilibrium for the PDP is independent of the ordering of issues. However, we view $X$ as a list because it makes it easier to refer to the individual elements of $X$.

\section{Optimal Agendas: Complete Information}

We first show how to find optimal agenda for the complete information setting and then for the incomplete information setting described in Section 3.2. For the complete information setting, Theorem 1 (Theorem 2) shows how to find $a$ 's ( $b$ 's) optimal agenda.

\footnotetext{
${ }^{2}$ This is situation occurs often: for the previous car dealer example, a buyer may have different preferences over the seat color and the car color, but the dealer may be indifferent between colors.

${ }^{3}$ Future work will deal with those situations where, for both $a$ and $b$, the weights are different for different issues.
} 
Then, for the incomplete information setting described in Section 3.2, Theorem 3 (Theorem 4) gives $a$ 's ( $b$ 's) optimal agenda. Theorem 5 gives the time complexity of computing the optimal agendas.

Theorem 1. In a setting where agent a has different weights for different issues and $b$ has the same weight for all $m$ issues, a's optimal agenda of size $g$ is a set of $g$ issues that are associated with the $g$ highest weights in $w^{a}$ (i.e., $A A^{g}=\{m-g+1, \ldots, m\}$ ).

Proof. Here $A A^{g}$ is obtained using BI. If an agenda is optimal for a for the last time period $t=n$, then by BI, it will be optimal for all previous ones. Consider the last time period $(t=n)$ for which two possibilities can arise: either a or $b$ could be the last mover. Consider first the case where a is the last mover. As per the equilibrium for the PDP, irrespective of the agenda, a gets a 100\% of all the shrunken pies. So, from among all possible agendas, its cumulative utility is maximized for the agenda $A A^{g}=\{m-g+1, \ldots, m\}$.

Now, consider the case where $b$ is the last mover. Here, as per the equilibrium for PDP, $b$ gets a 100\% of all the shrunken pies and a gets nothing (i.e., a's utility for $t=n$ is zero). Also, since b has equal weights for all the issues, its cumulative utility for $t=n$ is independent of the agenda. We therefore consider the previous time period $(t=n-1)$ for which it is a's turn to offer. As per the equilibrium for $t=n-1$, b's utility must be equal to its equilibrium utility for $t=n$. If $U^{b}$ denotes b's equilibrium utility for $t=n$, then in the equilibrium for $t=n-1, b$ must get $U^{b}$. Note that since $b$ has equal weights, $U^{b}$ is independent of the agenda. But a's utility depends on the agenda. Given $U^{b}$, a's equilibrium utility for $t=n-1$ (for an agenda $X \in A G^{g}$ ) will be $U_{X}^{a}=\left(\sum_{i=1}^{g} w_{X_{i}}^{a}\right)-U^{b}$. Then, a's optimal agenda is the one that maximizes $U_{X}^{a}$. Since all weights are positive, and the issues in $X=\{1, \ldots, g\}$ are in ascending order of $w_{i}^{a}$, $U_{X}^{a}$ is maximized when $X=\{m-g+1, \ldots, m\}$. Thus, $A A^{g}=\{m-g+1, \ldots, m\}$.

Regarding $b$ 's optimal agenda, one might think that $A B^{g}$ will be the set of $g$ issues that correspond to the $g$ lowest weights for $a$. But this is not so, because an agent's cumulative utility from an agenda depends not just on its weights but also on its equilibrium shares for the $g$ issues. The following example clarifies this point.

Example 1. Let $m=4, I=\{1,2,3,4\}, g=3, \delta=0.5, n=2, w^{a}=\{1,2,3,4\}$, $w^{b}=\{1,1,1,1\}$, and $b$ be first mover. There are four possible agendas of size $g=3$ : $\{1,2,3\},\{1,2,4\},\{1,3,4\}$, and $\{2,3,4\}$. For each of them, the agents' equilibrium utilities for $t=1$ (i.e., $U_{1}^{a}$ and $U_{1}^{b}$ ) are as given in Table 1. Agent $b$ 's utility $U_{1}^{b}$ is highest for the agenda $\{1,2,4\}$, and so $A B^{g}=\{1,2,4\}$ is $b$ 's optimal agenda. Likewise, $A A^{g}=\{2,3,4\}$.

As Example 1 shows, the optimal agenda may be different for different agents. But in many practical cases, only one of the two agents has a choice over the agenda. This is also the case in the car dealer example (outlined in Section 1) where the buyer can choose an agenda but not the seller.

We will now show how to find $b$ 's optimal agenda $A B^{g}$ using the following method. Initially, the optimal agenda is empty. Then, we add issues to it one by one using a greedy approach. For $1 \leq k \leq g$, let $A B_{k}^{g}$ denote the issue that is $b$ 's optimal choice at step $k$. Then, $b$ 's optimal agenda $A B^{k}$ of size $k$ is $A B^{k}=\cup_{i=1}^{k} A B_{i}^{g}$. 


\begin{tabular}{|c|c|c|c|}
\hline Agenda & $U_{1}^{a}$ & $U_{1}^{b}$ & $b$ 's Optimal Agenda? \\
\hline$\{2,3,4\}$ & 4.5 & 1.833 & No \\
$\{1,2,3\}$ & 3 & 2 & No \\
$\{\mathbf{1}, \mathbf{2}, \mathbf{4}\}$ & $\mathbf{3 . 5}$ & $\mathbf{2 . 1 2 5}$ & Yes \\
$\{1,3,4\}$ & 4 & 2 & No \\
\hline
\end{tabular}

Table 1. Agents' utilities (for $t=1$ ) for possible agendas.

Theorem 2. In a setting where agent a has different weights for different issues and $b$ has the same weight for all of them, b's optimal agenda $A B^{g}$ (for $g \geq 2$ ) is obtained as follows. Agent b's optimal choice for the first two issues is:

$$
A B_{1}^{g}=1 \quad \text { and } \quad A B_{2}^{g}=m .
$$

Then $A B_{k}^{g}(3 \leq k \leq g)$ is given by the following rule. If we let $\cup_{i=1}^{k-1} A B_{i}^{g}=\{1, \ldots, p, q, \ldots, m\}$ where $(1 \leq p<q \leq m)$ and $(p+m-q+1=k-1)$, then if

$$
\sum_{i=q}^{m} w_{i}^{a} \geq \delta \sum_{i=1}^{k-1} w_{A B_{i}^{g}}^{a}+\delta w_{p+1}^{a}
$$

then $A B_{k}^{g}=p+1$. Otherwise, if $\exists Z \in I-\cup_{i=1}^{k-1} A B_{i}^{g}$ such that

$$
w_{Z}^{a}+\sum_{i=q}^{m} w_{i}^{a} \geq \delta \sum_{i=1}^{k-1} w_{A B_{i}^{g}}^{a}+\delta w_{Z}^{a}
$$

then if

$$
\delta \sum_{i=1}^{k-1} w_{A B_{i}^{g}}^{a} \geq \sum_{r=q}^{m} w_{A B_{r}^{g}}^{a}
$$

then $A B_{k}^{g}=p+1$. Otherwise (i.e., Eq. 2 or 3 is false), $A B_{k}^{g}=q-1$.

Proof. An agent's optimal agenda problem exhibits the 'greedy choice property' and the 'optimal sub-structure property'. So a globally optimal solution can be found by making locally optimal choices. A problem has 'optimal substructure' if an optimal solution can be constructed from optimal solutions to its subproblems. An agent's optimal agenda problem has both these properties since all weights are positive and so an agent's cumulative utility is maximized if its utility from the individual issues is maximized. Given that b has equal weights for all the issues, b's cumulative utility is maximized if the issues are chosen such that its cumulative equilibrium share for them is the maximum over all possible agendas. So if $x_{X}^{b}$ denotes b's equilibrium share for agenda $X$, then $A B^{g}=\arg \max _{X \in A G^{g}} \sum_{i=1}^{g} x_{X_{i}}^{b}$ or $A B^{g}=\arg \min _{X \in A G^{g}} \sum_{i=1}^{g} x_{X_{i}}^{a}$ (since, $\left.x_{i}^{a}=1-x_{i}^{b}\right)$. To find $A B^{g}$, we will choose one issue at a time. At step $k(1 \leq k \leq g)$ we will choose $A B_{k}^{g}$ such that a's shares for the issues chosen thus far are minimized (relative to all possible agendas of size $k$ ). In more detail, this is done as follows. 
As before, if an agenda is optimal for $b$ for $t=n$, then by BI, it will be optimal for all previous time periods. Now, either a or b could be the last mover. If it is a, it gets a $100 \%$ of all the shrunken pies and $b$ gets zero utility. But if b is last mover, then it gets a 100\% of all shrunken pies and a gets nothing. So irrespective of who the last mover $i s$, $b$ 's utility for $t=n$ is independent of the agenda. Hence, we look at the previous time period $t=n-1$ to find $A B^{g}$. Consider the case where $b$ is the offering agent for $t=n-1$. If $x_{X}^{a}$ denotes a's equilibrium share for $t=n-1$, then $b$ 's optimal agenda for this time period is $A B^{g}=\arg \min _{X \in A G^{g}} \sum_{i=1}^{g} x_{X_{i}}^{a}$.

To begin, we show how to find the first and second issues (i.e., $A B_{1}^{g}$ and $A B_{2}^{g}$ ) that will be included in $A B^{g}$. Then, we will show how to find issues one by one (using a greedy approach) to include in $A B^{g}$ until the number of issues in it is $g$. Let the $m$ issues $\{1, \ldots, m\}$ be such that $w_{i}^{a} \leq w_{i+1}^{a}$ for $1 \leq i<m-1$. We decide what $A B_{1}^{g}$ and $A B_{2}^{g}$ should be on the basis of the relation between $w_{A B_{1}^{g}}^{a}$ and $w_{A B_{2}^{g}}^{a}$. Irrespective of what these two issues are, there are two ${ }^{4}$ possible relations between their weights: $w_{A B_{1}^{g}}^{a}<w_{A B_{2}^{g}}^{a}$ or else $w_{A B_{1}^{g}}^{a}>w_{A B_{2}^{g}}^{a}$. We consider each of these below.

For the case $w_{A B_{1}^{g}}^{a}<w_{A B_{2}^{g}}^{a}$ : At this stage, we know the relation between $w_{A B_{1}^{g}}^{a}$ and $w_{A B_{2}^{g}}^{a}$ but not the actual weights. Given this relation, as per the equilibrium for $t=n-1$ (see Section 3.2), $b$ will first allocate a share to a for the issue $A B_{2}^{g}$ and then for $A B_{1}^{g}$. This will be done such that a's cumulative utility (say $Y$ ) from $A B_{1}^{g}$ and $A B_{2}^{g}$ is equal to its cumulative utility for $t=n$ (i.e., $Y=\delta^{n-1} \sum_{i=1}^{2} w_{A B_{i}^{g}}^{a}$ ). When doing this allocation for $t=n-1$, one of two possible cases (C1.1 or C1.2) can arise:

C1.1 The entire utility $Y$ can be given to a from the issue $A B_{2}^{g}$ alone so a's share for $A B_{1}^{g}$ is zero, so we have:

$$
w_{A B_{2}^{g}}^{a} \delta^{n-2} \geq\left(w_{A B_{1}^{g}}^{a}+w_{A B_{2}^{g}}^{a}\right) \delta^{n-1}
$$

If $x_{A B_{2}^{g}}$ denotes a's equilibrium share for $A B_{2}^{g}$ for $t=n-1$, then as per Section 3.2, we have:

$$
x_{A B_{2}^{g}}^{a}=\left(w_{A B_{1}^{g}}^{a}+w_{A B_{2}^{g}}^{a}\right) \delta^{n-1} / w_{A B_{2}^{g}}^{a} \delta^{n-2}
$$

Clearly, it is optimal for $b$ to choose $A B_{1}^{g}$ and $A B_{2}^{g}$ such that $x_{A B_{2}^{g}}$ is minimized. In Equation 5, since $\delta$ is a constant, $x_{A B_{2}^{g}}^{a}$ is minimized when $w_{A B_{1}^{g}}^{a}$ is minimized and $w_{A B_{2}^{g}}^{a}$ is maximized. Thus, $A B_{1}^{g}$ is the issue with lowest weight in $w^{a}$, and $A B_{2}^{g}$, is the one with highest weight in $w^{a}$. So $A B_{1}^{g}=1$ and $A B_{2}^{g}=m$.

C1.2 The utility $Y$ cannot be given just from the issue $A B_{2}^{g}$ so a's share for $A B_{2}^{g}$ is a $100 \%$ of it and its share for $A B_{1}^{g}$ is non-zero, so Equation 4 is false. If $x_{A B_{1}^{a}}^{a}$ denotes a's equilibrium share for $A B_{1}^{g}$ for $t=n-1$, then as per Section 3.2, we have:

$$
\left(w_{A B_{1}^{g}}^{a} x_{A B_{1}^{g}}^{a}+w_{A B_{2}^{g}}^{a}\right) \delta^{n-2}=\left(w_{A B_{1}^{g}}^{a}+w_{A B_{2}^{g}}^{a}\right) \delta^{n-1}
$$

\footnotetext{
${ }^{4}$ For the case $w_{B^{1}}^{a}=w_{B^{2}}^{a}$, the agents' equilibrium shares are independent of $w_{A B_{1}^{g}}^{a}$ and $w_{A B_{1}^{g}}^{a}$. This can be verified by substituting $w_{A B_{1}^{g}}^{a}=w_{A B_{1}^{g}}^{a}$ in Equations 5 and 6 .
} 
and

$$
x_{A B_{1}^{g}}^{a}=\delta-\frac{w_{A B_{2}^{g}}^{a}}{w_{A B_{1}^{g}}^{a}}(1-\delta)
$$

Here, $x_{A B_{1}^{g}}^{a}$ is minimized when $w_{A B_{2}^{a}}^{a}$ is maximized and $w_{A B_{1}^{g}}^{a}$ is minimized. So $A B_{1}^{g}=1$ and $A B_{2}^{g}=m$. C1.2).

Thus, if $w_{A B_{1}^{g}}^{a}<w_{A B_{2}^{g}}^{a}$, then $A B_{1}^{g}=1$ and $A B_{2}^{g}=m$ for both cases (C1.1 and

For the case $w_{A B_{1}^{g}}^{a}>w_{A B_{2}^{g}}^{a}$ : This case is the same as the previous one, with $w_{A B_{1}^{g}}^{a}$ and $w_{A B_{2}^{g}}^{a}$ swapped.

Now, we will choose $A B_{3}^{g}$. Since $A B_{1}^{g}=1$ and $A B_{2}^{g}=m$, we have $w_{1}^{a}<w_{A B_{3}^{g}}^{a}<$ $w_{m}^{a}$. So, in the equilibrium for $t=n-1, b$ will first allocate to $a$, a share for the issue $A B_{2}^{g}$, then for $A B_{3}^{g}$, and finally for $A B_{1}^{g}$. In the equilibrium allocation for $t=n-1$, a's utility is $Y=\delta^{n-1} \sum_{i=1}^{3} w_{A B_{i}^{g}}^{a}$. We find a's equilibrium shares in this allocation by considering the following three possible cases (C3.1 that corresponds to Equation 1 being true, C3.2 that corresponds to Equation 1 being false and Equation 2 being true, or C3.3 that corresponds to Equations 1 and 2 being false) that can arise:

C3.1 The entire utility $Y$ can be given to a from the issue $A B_{2}^{g}$ alone so a's share for $A B_{1}^{g}$ and $A B_{3}^{g}$ is each zero. So we have:

$$
w_{A B_{2}^{g}}^{a} \delta^{n-2} \geq \sum_{i=1}^{3} w_{A B_{i}^{g}}^{a} \delta^{n-1}
$$

If $x_{A B_{2}^{g}}$ denotes a's equilibrium share for $A B_{2}^{g}$ for $t=n-1$, then as per Section 3.2, we have:

$$
x_{A B_{2}^{g}}^{a}=\left(\sum_{i=1}^{3} w_{A B_{i}^{g}}^{a}\right) \delta^{n-1} / w_{A B_{2}^{g}}^{a} \delta^{n-2}
$$

Here, $x_{A B_{2}^{g}}$ is minimized by choosing as $A B_{3}^{g}$ the issue with lowest weight from the remaining issues in $I-\left\{A B_{1}^{g} \cup A B_{2}^{g}\right\}$. This gives $A B_{3}^{g}=2$. Moreover, Equation 7 is true iff $A B_{3}^{g}=2$ because, in $I-\left\{A B_{1}^{g} \cup A B_{2}^{g}\right\}, 2$ is the issue with least weight. C3.2 The utility $Y$ cannot be given just from $A B_{2}^{g}$ but can be given from $A B_{2}^{g}$ and $A B_{3}^{g}$. So a's share for $A B_{2}^{g}$ is a hundred percent of it and its share for $A B_{3}^{g}$ is non-zero, i.e., Equation 7 is false $\forall A B_{3}^{g} \in I-\left\{A B_{1}^{g} \cup A B_{2}^{g}\right\}$ and $\exists A B_{3}^{g} \in$ $I-\left\{A B_{1}^{g} \cup A B_{2}^{g}\right\}$ s.t.:

$$
\left(w_{A B_{2}^{g}}^{a}+w_{A B_{3}^{g}}^{a}\right) \delta^{n-2} \geq \sum_{i=1}^{3} w_{A B_{i}^{g}}^{a} \delta^{n-1}
$$

If $x_{A B_{3}^{g}}^{a}$ denotes a's equilibrium share for $A B_{3}^{g}$ for $t=n-1$, then as per Section 3.2, we have:

$$
\left(w_{A B_{3}^{g}}^{a} x_{A B_{3}^{g}}^{a}+w_{A B_{2}^{g}}^{a}\right) \delta^{n-2}=\sum_{i=1}^{3} w_{A B_{i}^{g}}^{a} \delta^{n-1}
$$


Solving the above equation for $x_{A B_{3}^{g}}^{a}$, we get:

$$
x_{A B_{3}^{g}}^{a}=\frac{\sum_{i=1}^{2} \delta w_{A B_{i}^{g}}^{a}-w_{A B_{2}^{g}}^{a}}{w_{A B_{3}^{g}}^{a}}+\delta
$$

Here, if Equation 3 is true, then $x_{A B_{3}^{g}}^{a}$ is minimized if $w_{A B_{3}^{g}}^{a}$ is maximized. So $A B_{3}^{g}$ must be the issue with lowest weight from the remaining issues, or $A B_{3}^{g}=2$. But if Equation 3 is false, $x_{A B_{3}^{g}}$ is minimized when $A B_{3}^{g}=m-1$.

C3.3 The entire utility $Y$ cannot be given from $A B_{2}^{g}$ and $A B_{3}^{g}$ so a's share for each of $A B_{2}^{g}$ and $A B_{3}^{g}$ is a hundred percent and its share for $A B_{1}^{g}$ is non-zero, i.e., Equations 7 and 9 are false $\forall A B_{3}^{g} \in I-\left\{A B_{1}^{g} \cup A B_{2}^{g}\right\}$, so:

$$
\left(\sum_{i=1}^{3} w_{A B_{i}^{g}}^{a}\right) \delta^{n-2} \geq \sum_{i=1}^{3} w_{A B_{i}^{g}}^{a} \delta^{n-1}
$$

If $x_{A B_{1}^{g}}^{a}$ denotes a's equilibrium share for $A B_{1}^{g}$ for $t=n-1$, then as per Section 3.2, we have:

$$
\left(w_{A B_{1}^{g}}^{a} x_{A B_{1}^{g}}^{a}+w_{A B_{2}^{g}}^{a}+w_{A B_{3}^{g}}^{a} \delta^{n-2}=\sum_{i=1}^{3} w_{A B_{i}^{g}}^{a} \delta^{n-1}\right.
$$

Solving the above equation for $x_{A B_{1}^{g}}^{a}$, we get:

$$
x_{A B_{1}^{g}}^{a}=\left(\delta w_{A B_{1}^{g}}^{a}+\delta w_{A B_{2}^{g}}^{a}-w_{A B_{2}^{g}}^{a}-w_{A B_{3}^{g}}^{a}(1-\delta)\right) / w_{A B_{1}^{g}}^{a}
$$

Here, $x_{A B_{1}^{g}}^{a}$ is minimized when $A B_{3}^{g}=m-1$.

Thus, for the case C3.1, $A B_{3}^{g}=2$. For C3.2, $A B_{3}^{g}=2$ or $A B_{3}^{g}=m-1$ (depending on Equation 3). And for $C 3.3 A B_{3}^{g}=m-1$.

In general, to decide what to choose as $A B_{k}^{g}(4 \leq k \leq g)$, we find a's equilibrium allocation for each of the $k$ issues (chosen thus far) for $t=n-1$. At stage $k$,we already have the optimal agenda of size $k-1$, i.e., $\cup_{i=1}^{k-1} A B_{i}^{g}$. Also, we know that for stage $i$, $(1 \leq i \leq k-1)$ b's optimal choice is either the issue with highest weight for a or else the one with the lowest weight from the remaining issues $I-\left\{\cup_{j=1}^{i-1} A B_{j}^{g}\right\}$. Hence, as mentioned in the statement of this theorem, we let $\cup_{i=1}^{k-1} A B_{i}^{g}=\{1, \ldots, p, q, \ldots, m\}$ where $(1 \leq p<q \leq m)$ and $(p+m-q+1=k-1)$. This implies that $w_{p}^{a}<w_{A B_{k}^{g}}^{a}<$ $w_{q}^{a}$. Also, as before, the equilibrium allocation for $k$ issues for $t=n-1$ must give a a utility of $Y=\delta^{n-1} \sum_{i=1}^{k} w_{A B_{i}^{g}}^{a}$. We find a's equilibrium shares in this allocation by considering the following three possible cases (CK.1 that corresponds to Equation 1 being true, CK.2 that corresponds to Equation 1 being false and Equation 2 being true, or CK.3 that corresponds to Equations 1 and 2 being false) that can arise: 
CK.1 The cumulative utility $Y$ can be given to a just from the pies in $\{j, \ldots, m\}$ (where $q \leq j \leq m$ ) so we have ${ }^{5}$ :

$$
\delta^{n-2} \sum_{r=j}^{m} w_{\alpha_{r}}^{a} \geq \delta^{n-1} \sum_{i=1}^{k} w_{A B_{i}^{g}}^{a}
$$

If $x_{A B_{k}^{g}}^{a}$ denotes a's equilibrium share for $A B_{k}^{g}$ for $t=n-1$, then as per Section 3.2, we have:

$$
x_{j}^{a}=\left(\delta \sum_{i=1}^{k} w_{A B_{i}^{g}}^{a}-\sum_{r=j+1}^{m} w_{r}^{a}\right) / w_{j}^{a}
$$

Here, $x_{j}^{a}$ is minimized if $w_{A B_{k}^{g}}^{a}$ is minimized. So b's optimal choice for $A B_{k}^{g}$ is the issue with lowest weight from the remaining issues $I-\left\{\cup_{i=1}^{k-1} A B_{i}^{g}\right\}$, i.e., $A B_{k}^{g}=$ $p+1$. Moreover Equation 11 is true iff $A B_{k}^{g}=p+1$ because, in $I-\left\{\cup_{i=1}^{k-1} A B_{i}^{g}\right\}$, $p+1$ is the issue with least weight.

CK.2 The entire utility $Y$ cannot be given just from the pies in $\{q, \ldots, m\}$ but can be given from $\{q, \ldots, m\}$ together with $A B_{k}^{g}$, i.e., $\exists A B_{k}^{g} \in I-\left\{\cup_{i=1}^{k-1} A B_{i}^{g}\right\}$ such that:

$$
\begin{array}{r}
\delta^{n-2} \sum_{r=q}^{m} w_{r}^{a}<\delta^{n-1} \sum_{i=1}^{k} w_{A B_{i}^{g}}^{a}, \quad \text { and } \\
\delta^{n-1}\left(w_{A B_{k}^{g}}^{a} x_{A B_{k}^{g}}^{a}+\sum_{r=q}^{m} w_{r}^{a}\right) \geq \delta^{n-2} \sum_{i=1}^{k} w_{A B_{i}^{g}}^{a}
\end{array}
$$

If $x_{A B_{k}^{g}}^{a}$ denotes a's equilibrium share for $A B_{k}^{g}$ for $t=n-1$, then as per Section 3.2, we have:

$$
x_{A B_{k}^{g}}^{a}=\frac{\delta \sum_{i=1}^{k-1} w_{A B_{i}^{g}}^{a}-\sum_{r=q}^{m} w_{r}^{a}}{w_{A B_{k}^{a}}^{a}}+\delta
$$

Here, if Equation 3 is true, then $x_{A B_{k}^{a}}^{a}$ is minimized if $w_{A B_{k}^{g}}^{a}$ is maximized, i.e., $A B_{k}^{g}=p+1$. But if Equation 3 is false, $x_{A B_{k}^{g}}^{a}$ is minimized if $w_{A B_{k}^{g}}^{a}$ is minimized, i.e., $A B_{k}^{g}=q-1$.

CK.3 The cumulative utility $Y$ cannot be given just from the pies in $\left\{A B_{k}^{g}, q, \ldots, m\right\}$ but can be given from $\left\{j, \ldots, r, A B_{k}^{g}, q, \ldots, m\right\}$ where $1 \leq j \leq p$, so we have:

$$
\delta^{n-2}\left(w_{A B_{k}^{g}}^{a}+\sum_{r=q}^{m} w_{r}^{a}\right)<\delta^{n-1} \sum_{i=1}^{k} w_{A B_{i}^{g}}^{a}, \quad \text { and }
$$

\footnotetext{
${ }^{5}$ Here, $a$ 's share for each of the pies $j+1, \ldots, m$ is one, for pie $j$ it is $x_{j}^{a}$, and for each of the pies $1, \ldots, j-1$, is zero.
} 


$$
\delta^{n-1}\left(\sum_{r=j}^{p} w_{r}^{a}+w_{A B_{k}^{g}}^{a}+\sum_{r=q}^{m} w_{r}^{a}\right) \geq \delta^{n-2} \sum_{i=1}^{k} w_{A B_{i}^{g}}^{a}
$$

If $x_{A B_{k}^{g}}$ denotes a's equilibrium share for $A B_{k}^{g}$ for $t=n-1$, then as per Section 3.2, we have:

$$
x_{j}^{a}=\left(\delta \sum_{i=1}^{k} w_{A B_{i}^{g}}^{a}-\sum_{r=q}^{m} w_{r}^{a}-w_{A B_{k}^{g}}^{a}-\sum_{r=j+1}^{p} w_{r}^{a}\right) / w_{j}^{a}
$$

Here $x_{j}^{a}$ is minimized when $w_{A B_{k}^{g}}^{a}$ is maximized. So $A B_{k}^{g}$ must be the issue with highest weight from the remaining issues $I-\left\{\cup_{i=1}^{k-1} A B_{i}^{g}\right\}$, i.e., $A B_{k}^{g}=q-1$.

Thus, for the case CK.1, $A B_{k}^{g}=p+1$. For CK.2, $A B_{k}^{g}=p+1$ or $A B_{k}^{g}=q-1$ (depending on Equation 3). And for CK.3, $A B_{k}^{g}=q-1$.

In the same way, we can obtain b's optimal agenda for the case where a is the offering agent at $t=n-1$.

\section{Optimal Agendas: Incomplete Information}

We will now show how to find optimal agendas for the incomplete information setting of Section 3.2. To this end, Theorem 3 gives $a$ 's optimal agenda and Theorem 4 that for $b$. Finally, Theorem 5 gives the time complexity of computing these optimal agendas. Let the $m$ issues $\{1, \ldots, m\}$ be such that: $e w_{1}^{a} \leq e w_{2}^{a} \leq \ldots \leq e w_{m}^{a}$. Here, agent $a$ has different expected weights for different issues, but $b$ has the same expected weight for all the $m$ issues.

Theorem 3. In a setting where agent a has different expected weights for different issues but, for b, the set of possible weights for an issue and the associated probabilities are the same for all the $m$ issues, agent a's optimal agenda of size $g$ is a set of $g$ issues that are associated with the $g$ highest expected weights (i.e., $A A^{g}=$ $\{m-g+1, \ldots, m\}$ ).

Proof. As Theorem 1, with weights replaced with corresponding expected weights and utilities with corresponding expected utilities.

Theorem 4. In a setting where agent a has different expected weights for different issues but, for $b$, the set of possible weights for an issue and the associated probabilities are the same for all the $m$ issues, b's optimal agenda $A B^{g}($ for $g \geq 2)$ is obtained as in Theorem 2 with agent's weights replaced with corresponding expected weights, and utilities replaced with corresponding expected utilities.

Proof. As Theorem 2, with weights replaced with corresponding expected weights and utilities with corresponding expected utilities.

Theorem 5. For the complete information setting, the time taken to compute $A A^{g}$ is $\mathcal{O}(g)$ and to compute $A B^{g}$ is $\mathcal{O}\left(m g^{2}\right)$. For the incomplete information setting, the time to compute $A A^{g}$ is $\mathcal{O}(T g)$ and to compute $A B^{g}$ is $\mathcal{O}\left(T m g^{2}\right)$. 
Proof. For the complete information setting, as per Theorem $1, A A^{g}$ is the set of $g$ issues with highest weight in $w^{a}$. We therefore need to choose the last $g$ issues from $\alpha$ which takes time $\mathcal{O}(g)$. For $A B^{g}$, as per Theorem 2, determining $A B_{k}^{g}(1 \leq k \leq$ g) requires evaluating the conditions in Equations 1, 2, and 3. Consider Equation 2 which requires one comparison between $w_{Z}^{a}+\sum_{i=q}^{m} w_{i}^{a}$ and $\delta \sum_{i=1}^{k-1} w_{A B_{i}^{g}}^{a}+\delta w_{Z}^{a}$. Computation of these two terms to compare requires no more than $k$ additions because the summation variable $i$ varies between 1 and $k$. Also, $Z$ can vary at most between 1 and $m$. So finding $A B_{k}^{g}$ takes $\mathcal{O}(m k)$ time, and to find $A B_{k}^{g}$ for $1 \leq k \leq g$, it takes $\sum_{k=1}^{g} \mathcal{O}(m k)=\mathcal{O}\left(m g^{2}\right)$ time. Note that we considered only Equation 2 because the time to evaluate the condition in Equations 1 or 3 is no more than the time taken to evaluate the condition in Equation 2.

It follows that, for the incomplete information setting, if there are $T$ possible utility function pairs, the time to compute $A A^{g}$ is $\mathcal{O}(T g)$ and to compute $A B^{g}$ is $\mathcal{O}\left(T m g^{2}\right)$.

\section{Conclusions and Future Work}

This paper presented polynomial time methods for finding each agent's optimal agenda for the PDP. The polynomial time complexity of our methods makes it easy for automating the process of choosing an agent's optimal agenda, and thereby reducing human involvement during negotiation.

Possible avenues for future research include extending the current analysis to scenarios where both agents have different weights for different issues. Also, this paper focussed on one specific incomplete information setting. In future, we will extend this analysis to other possible incomplete information settings. 


\section{Bibliography}

[1] M. Bac and H. Raff. Issue-by-issue negotiations: the role of information and time preference. Games and Economic Behavior, 13:125-134, 1996.

[2] L. A. Busch and I. J. Horstman. A comment on issue-by-issue negotiations. Games and Economic Behavior, 19:144-148, 1997.

[3] T. H. Cormen, C. E. Leiserson, R. L Rivest, and C. Stein. An introduction to algorithms. The MIT Press, Cambridge, Massachusetts, 2003.

[4] S. S. Fatima, M. Wooldridge, and N. R. Jennings. Optimal agendas for multiissue negotiation. In Proceedings of the Second International Conference on Autonomous Agents and Multi-Agent Systems, pages 129-136, Melbourne, Australia, July 2003.

[5] S. S. Fatima, M. Wooldridge, and N. R. Jennings. An agenda based framework for multi-issue negotiation. Artificial Intelligence Journal, 152(1):1-45, 2004.

[6] S. S. Fatima, M. Wooldridge, and N. R. Jennings. Multi-issue negotiation with deadlines. Journal of Artificial Intelligence Research, 27:381-417, 2006.

[7] C. Fershtman. The importance of the agenda in bargaining. Games and Economic Behavior, 2:224-238, 1990.

[8] C. Fershtman. A note on multi-issue two-sided bargaining: bilateral procedures. Games and Economic Behavior, 30:216-227, 2000.

[9] D. Fudenberg and J. Tirole. Sequential bargaining with incomplete information. Review of Economic Studies, 50:221-247, 1983.

[10] S. Kraus. Strategic negotiation in multi-agent environments. The MIT Press, Cambridge, Massachusetts, 2001.

[11] D. A. Lax and J. K. Sebenius. Dealcrafting: The substance of three dimensional negotiations. Negotiation Journal, 18(1):5-28, 2002.

[12] M. J. Osborne and A. Rubinstein. A Course in Game Theory. The MIT Press, 1994.

[13] J. S. Rosenschein and G. Zlotkin. Rules of Encounter. MIT Press, 1994.

[14] J. K. Sebenius. Negotiation arithmetic: Adding and subtracting issues and parties. International Organization, 37(2):281-316, 1983. 